\title{
APPROXIMATION AND EXTENSION OF CONTINUOUS FUNCTIONS
}

\author{
SALVADOR HERNÁNDEZ-MUÑOZ
}

(Received 19 February 1991; revised 1 February 1992)

Communicated by J. H. Rubinstein

\begin{abstract}
In this paper we study the approximation of vector valued continuous functions defined on a topological space and we apply this study to different problems. Thus we give a new proof of Machado's Theorem. Also we get a short proof of a Theorem of Katětov and we prove a generalization of Tietze's Extension Theorem for vector-valued continuous functions, thereby solving a question left open by Blair.
\end{abstract}

1991 Mathematics subject classification (Amer. Math. Soc.): primary 54C20, 54C30, 54D60, 46E25; secondary 54C45, 54C99, 46E15.

In 1977 Machado stated a theorem on approximation of vector-valued continuous functions defined on a compact space which generalized a celebrated theorem on approximation of continuous functions given by Bishop (see [2] and [8]). The original proof of Machado's theorem was elementary but long (a self-contained and detailed version of this result has been given by Burckel in [4]). Recently, Ransford [10] and Edwards [5] have published short proofs of Machado's theorem. Here we follow the ideas outlined in [8] by Machado, in order to obtain results on approximation of continuous functions in more general situations. Thus we can give a proof of Machado's theorem which eliminates some technical calculations appearing in [8] and [4] (see [4, Lemma 1 and Lemma 2]). Also we get a very short proof of a Theorem of Katětov [7] stating that paracompactness implies realcompactness when the cardinality is non-measurable. Finally, a generalization of Tietze's Extension Theorem for vector-valued continuous functions is proved by using this approach, thereby solving a question by Blair.

We use $\mathbb{N}$ (respectively $\mathbb{R}, \mathbb{C}$ ) to denote the set of all natural numbers (respectively real, complex numbers). Throughout, $X$ is a nonvoid completely regular Hausdorff

(C) 1994 Australian Mathematical Society 0263-6115/94 \$A2.00+0.00 
space, and $E$ is a real or complex Banach space. We use $C(X, E)$ (respectively $C^{*}(X, E)$ ) to denote the set of all continuous maps (respectively with precompact range) of $X$ into $E$. If $e \in E$ and $A \subseteq E$ we denote by $d(e, A)$ the number $\inf \{\|e-a\|: a \in A\}$, and we denote by $B_{\epsilon}(A)$ the set $\{e: e \in E, d(e, A)<\epsilon\}$ (here \|\| denotes the norm in $E$ ). When $B$ is a subset of a topological space we denote by cl $B$ closure of $B$ in that topological space.

We endow $C(X, E)$ with the topology of uniform convergence, that is, for every $F \in C(X, E)$ a neighbourhood base for $F$ is defined by sets of the form $N_{\epsilon}(F)=$ $\{G: G \in \mathscr{C}(X, E),\|F(x)-G(x)\|<\epsilon$ for all $x \in X\}$, with $\epsilon$ being an arbitrary positive real number. If $F \in C(X, E), \mathscr{G} \subseteq C(X, E)$ and $S \subseteq X$ we define $d_{S}(F, \mathscr{G})=\inf \{\sup \{\|F(x)-G(x)\|: x \in S\}: G \in \mathscr{G}\}$ (notice that $d(F, \mathscr{G})$ may be $+\infty)$. We write $C(X)$ for $C(X, \mathbb{R})$.

For $S \subseteq X$ and $F$ a function on $X$ we write $\left.F\right|_{S}$ for the restriction of $F$ to $S$. If $\mathscr{G} \subseteq C(X, E)$ then $\left.\mathscr{G}\right|_{S}=\left\{\left.G\right|_{s}: G \in \mathscr{G}\right\}$. Let $\mathscr{A} \subseteq C(X)$ and $\mathscr{G} \subseteq C(X, E)$. If $\alpha$ is a cardinal number, we denote by $\langle\mathscr{G}, \mathscr{A}\rangle_{\alpha}$ the set of all functions defined by sums of the form $\sum_{i \in I} h_{i} G_{i}$ with $|I|<\alpha,\left\{h_{i}: i \in I\right\} \subseteq \mathscr{A},\left\{G_{i}: i \in I\right\} \subseteq \mathscr{G}$ and $\left\{\operatorname{supp} h_{i}: i \in I\right\}$ is a locally finite family of sets on $X$, here supp $h_{i}$ denotes the support of $h_{i}$ on $X$. It is clear that if $\mathscr{G}$ is a module over $\mathscr{A}$ with respect to pointwise multiplication of functions, then $\langle\mathscr{G}, \mathscr{A}\rangle_{\aleph_{0}} \subseteq \mathscr{G}$ and if $\mathscr{A}$ is a subalgebra of $C(X)$ which contains the unit then the inclusion becomes an equality. We write $\langle\mathscr{G}, \mathscr{A}\rangle$ when no restrictions on cardinality are imposed on the index set of the above sums.

When $\mathscr{A}$ is contained in $C(X)$ or $C(X, \mathbb{C})$, a nonvoid subset $S$ of $X$ is called an $\mathscr{A}$-antisymmetric set if whenever $f$ is an element of $\mathscr{A}$ and the restriction of $f$ to $S$ is real-valued, then the restriction of $f$ to $S$ is constant. Thus, for real algebras $\mathscr{A}$, an antisymmetric set is the same as a set of constancy for the algebra. If we denote by $\mathscr{A}(X)$ the family of all maximal $\mathscr{A}$-antisymmetric sets of $X$, then $\mathscr{A}(X)$ defines a unique decomposition of $X$ into pairwise disjoint, nonvoid, maximal $\mathscr{A}$ antisymmetric sets, which are closed when $\mathscr{A}$ consists of continuous functions only (see [4]).

If $\mathscr{A} \subseteq C(X), \mathscr{G} \subseteq C(X, E)$ and $\alpha$ is a cardinal number, we say $\mathscr{A} \alpha$-refines $\mathscr{G}$ when for every open cover $\mathscr{U}$ of $X$ of the form $\mathscr{U}=\left\{G_{i}^{-1}\left(B_{\epsilon}\left\{G_{i}\left(S_{i}\right)\right\}\right): G_{i} \in \mathscr{G}, S_{i} \in\right.$ $\mathscr{A}(X), i \in I\}$ there is a locally finite partition of unity $\left\{h_{j}: j \in J\right\} \subseteq \mathscr{A}$ which is subordinate to the cover $\mathscr{U}$, with $h_{j} \geq 0$ for all $j \in J$ and $|J|<\alpha$. We say that $\mathscr{A}$ refines $\mathscr{G}$ when there is no restriction on the cardinality of $J$.

We shall begin this paper by stating some technical lemmas.

LemMA 1. Let $\mathscr{A} \subseteq C(X), \mathscr{G} \subseteq C(X, E)$ and $F \in C(X, E)$.

(i) If $\mathscr{A} \alpha$-refines the family $\{F-G: G \in \mathscr{G}\}$, then $d_{X}\left(F,\langle\mathscr{G}, \mathscr{A}\rangle_{\alpha}\right) \leq$ $\sup \left\{d_{S}(F, \mathscr{G}): S \in \mathscr{A}(X)\right\}$.

(ii) Suppose that $\mathscr{A}(X)$ consists only of singletons and $\mathscr{G}$ contains all constant functions. If $\mathscr{A} \alpha$-refines $\{F\}$ then $d_{X}\left(F,\langle\mathscr{G}, \mathscr{A}\rangle_{\alpha}\right)=0$. 
PROOF.

(i) Set $r=\sup \left\{d_{S}(F, \mathscr{G}): S \in \mathscr{A}(X)\right\}$. If $r=+\infty$, the proof of (i) is trivial, so we can assume for the rest of the proof that $r<\infty$. Let $\epsilon$ be a positive real number. For every $S \in \mathscr{A}(X)$ there is $G_{S}$ in $\mathscr{G}$ such that $d_{S}\left(F, G_{s}\right)<r+\epsilon$. Set $U_{\epsilon}(S)=$ $\left(F-G_{S}\right)^{-1}\left(B_{\epsilon}\left\{\left(F-G_{S}\right)(S)\right\}\right)$ and consider the cover $\mathscr{U}=\left\{U_{\epsilon}(S): S \in \mathscr{A}(X)\right\}$. By hypothesis, there is a locally finite partition of unity $\left\{h_{i}: i \in I\right\} \subseteq \mathscr{A}$ with $|I|<\alpha$, which is subordinate to $\mathscr{U}$. For every $i \in I$, we take one $S_{i} \in \mathscr{A}(X)$ such that supp $h_{i} \subseteq U_{\epsilon}\left(S_{i}\right)$ and define $H=\sum_{i \in I} h_{i} \cdot G_{S_{i}}$. We have that $\|F(x)-H(x)\| \leq$ $\sum_{i \in I} h_{i}(x) .\left\|F(x)-G_{S_{i}}(x)\right\|<r+2 \epsilon$ for all $x \in X$ (note that if $x \in U_{\epsilon}(S)$ then there is $y \in S$ such that $\left|\left\|F(x)-G_{S}(x)\right\|-\left\|F(y)-G_{S}(y)\right\|\right|<\epsilon$; thus $\left\|F(x)-G_{S}(x)\right\|<$ $r+2 \epsilon)$, and so $d_{X}(F, H) \leq r+2 \epsilon$. Therefore $d_{X}\left(F,(\mathscr{G}, \mathscr{A}\rangle_{\alpha}\right) \leq r$.

(ii) Let $\epsilon$ be a positive real number. For every $x \in X$ set $U_{\epsilon}(x)=F^{-1}\left(B_{\epsilon}(F(x))\right)$ and consider the cover $\mathscr{U}=\left\{U_{\epsilon}(x): x \in X\right\}$. By hypothesis, there is a locally finite partition of unity $\left\{h_{i}: i \in I\right\}$ which is subordinate to $\mathscr{U}$ with $|I|<\alpha$. For every $i \in I$, we take one $x_{i} \in X$ such that supp $h_{i} \subseteq U_{\epsilon}\left(x_{i}\right)$ and define $H=\sum_{i \in I} h_{i} . F\left(x_{i}\right)$. We argue as in (i) that $d_{X}(F, H) \leq \epsilon$ and this completes the proof.

LEMMA 2. Let $\mathscr{A} \subseteq C^{*}(X)$ and $\mathscr{G} \subseteq C^{*}(X, E)$. Denote by $\mathrm{cl}_{u} \mathscr{A}$ the closure of $\mathscr{A}$ in $C^{*}(X)$ with the topology of uniform convergence. Then $d_{X}\left(F,\langle\mathscr{G}, A\rangle_{\aleph_{0}}\right)=$ $d_{X}\left(F,\left\langle\mathscr{G}, \mathrm{cl}_{u} \mathscr{A}\right\rangle_{\aleph_{0}}\right)$.

PROOF. Let $\left.r=d_{X}\left(F,<\mathscr{G}, \mathrm{cl}_{u} \mathscr{A}\right\rangle_{\aleph_{0}}\right)$ and let $e$ be a positive real number. Take $H=\sum_{i=1}^{n} h_{i} \cdot G_{i}$, with $h_{i} \in \operatorname{cl}_{u} \mathscr{A}$ and $G_{i} \in \mathscr{G}$, such that $d_{X}(F, H)<r+\epsilon$. Now let $K$ be a positive real number with $\left\|G_{i}(x)\right\| \leq K$ for all $x \in X$ and $i \in\{1, \ldots, n\}$, and take $g_{i} \in \mathscr{A}$ such that $d_{X}\left(h_{i}, g_{i}\right)<\epsilon / n K$. Then $L=\sum_{i=1}^{n} g_{i} \cdot G_{i}$ belongs to $\langle\mathscr{G}, \mathscr{A}\rangle_{\aleph_{0}}$ and $\|F(x)-L(x)\| \leq\|F(x)-H(x)\|+\|H(x)-L(x)\|<r+2 \epsilon$ for all $x \in X$. This proves that $d_{X}\left(F,\langle\mathscr{G}, \mathscr{A}\rangle_{\aleph_{0}}\right) \leq r$. Since it is obvious that $d_{X}\left(F,\langle\mathscr{G}, \mathscr{A}\rangle_{\aleph_{0}} h_{0}\right) \geq r$, the proof is complete.

Now we are going to apply the lemmas above to functions with precompact range. In order to do this we shall need another definition and lemma.

Let $F \in C(X, E)$ and $\mathscr{A} \subseteq C(X)$, we say that $\mathscr{A}$ separates $F$ (terminology inspired by [10]) when for all real numbers $r$ and $t$ with $0<r<t$, and for every $S \in \mathscr{A}(X)$, there is a function $h \in \mathscr{A}$ and a real constant $p$ such that $h\left(F^{-1}\left(\operatorname{cl}\left(B_{r}(\{F(S)\})\right)\right) \geq\right.$ $p>0$ and $h\left(X \backslash F^{-1}\left(B_{t}(\{F(S)\})\right)\right)=0$.

LEMMA 3. Let $\mathscr{A}$ be a uniformly closed subalgebra of $C^{*}(X)$ which contains the unit and let $F \in C^{*}(X, E)$. If $\mathscr{A}$ separates $F$ then $\mathscr{A} \aleph_{0}$-refines $\{F\}$.

Proof. Define $U_{r}(S)=F^{-1}\left(B_{r}(\{F(S)\})\right)$ and $\bar{U}_{r}(S)=F^{-1}\left(\operatorname{cl} B_{r}(\{F(S)\})\right)$ for $S \in \mathscr{A}(X)$ and $r$ a positive real number. Let $\epsilon$ be a positive real number and consider 
the covers of $X, \mathscr{U}=\left\{U_{\epsilon}(S): S \in \mathscr{A}(X)\right\}$ and $\mathscr{V}=\left\{U_{\epsilon / 2}(S): S \in \mathscr{A}(X)\right\}$. Since $\mathrm{F}$ has precompact range, $\mathscr{V}$ has a finite subcover $\left\{U_{\epsilon / 2}\left(S_{1}\right), \ldots, U_{\epsilon / 2}\left(S_{n}\right)\right\}$. For every $i$ with $1 \leq i \leq n$ take $g_{i} \in \mathscr{A}$ such that $g_{i}\left(\bar{U}_{\epsilon / 2}\left(S_{i}\right)\right) \geq r_{i}>0$, and $g_{i}\left(X-U_{2 \epsilon / 3}\left(S_{i}\right)\right)=0$, being $g_{i} \geq 0$. It is clear that $g=g_{1}+\ldots+g_{n}$ belongs to $\mathscr{A}$ and $g \geq p>0$ for some $p \in \mathbb{R}$. Since $\mathscr{A}$ is closed under uniform convergence and contains the unit, we have $1 / g \in \mathscr{A}$. If we define $h_{i}=g_{i} / g$, then $\left\{h_{i}: 1 \leq i \leq n\right\}$ is a partition of unity subordinate to $\mathscr{A}$. And, as $\epsilon$ was taken as an arbitrary positive real number, we deduce that $\mathscr{A} \aleph_{0}$-refines $\{F\}$.

Let us see some applications of the lemmas above.

THEOREM 4. Let $\mathscr{A} \subseteq C^{*}(X), \mathscr{G} \subseteq C^{*}(X, E)$ be such that each element of $\mathscr{A}(X)$ is a singleton and $\mathscr{G}$ contains the constant functions. If $\mathscr{A}$ separates $F \in C^{*}(X, E)$, then $d_{X}\left(F,\langle\mathscr{G}, \mathscr{A}\rangle_{\aleph_{0}}\right)=0$.

PROOF. This is clear by applying Lemma 1 (i), Lemma 2 and Lemma 3.

THEOREM 5. Let $X$ be a compact space, $\mathscr{A}$ a subalgebra of $C(X)$ which contains the unit and $\mathscr{G}$ a vector subspace of $C(X, E)$. If $F \in C(X, E)$, then there is $S \in \mathscr{A}(X)$ such that $d_{X}\left(F,\langle\mathscr{G}, \mathscr{A}\rangle_{\aleph_{0}}\right)=d_{S}(F, \mathscr{G})$.

ProOF. By Lemma 2 we can suppose that $\mathscr{A}$ is a uniformly closed subalgebra of $C(X)$, since $\mathscr{A}$ and $\operatorname{cl}_{u} \mathscr{A}$ have the same antisymmetric sets. Let us see that $\mathscr{A}$ $\aleph_{0}$-refines to $\{F-G: G \in \mathscr{G}\}$. Let $\epsilon$ be a positive real number and consider the open cover $\mathscr{U}$ of $X$, defined by $\mathscr{U}=\left\{\left(F-G_{i}\right)^{-1}\left(B_{\epsilon}\left(\left\{\left(F-G_{i}\right)\left(S_{i}\right)\right\}\right)\right): G_{i} \in \mathscr{G}, S_{i} \in\right.$ $\mathscr{A}(X), i \in I\}$. Set $U_{i}=(F-G)^{-1}\left(B_{\epsilon}\left(\left\{\left(F-G_{i}\right)\left(S_{i}\right)\right\}\right)\right)$; for every $i \in I$ and for all $y \in X \backslash U_{i}$, there is $g_{y} \in \mathscr{A}$ such that $g_{y}(S) \neq g_{y}(y)$ and, since $\mathscr{A}$ is a subalgebra of $C(X)$ which contains the unit, we can suppose that $g_{y}(S)=1, g_{y}(y)=0$ and $g_{y} \geq 0$ on $X$. On the other hand, as $\mathscr{A}$ is closed under uniform convergence, we deduce that $\mathscr{A}$ is a lattice of functions. Thus, by applying a compactness argument, we conclude that there is $g_{i} \in \mathscr{A}$ with $g_{i}\left(S_{i}\right)=1, g_{i}\left(X \backslash U_{i}\right)=0$ and $0 \leq g_{i} \leq 1$. Consider the cover of $X$ defined by $\left\{g_{i}^{-1}((1 / 2,1]): i \in I\right\}$; it has, by compactness, a finite subcover, $\left\{g_{j}^{-1}((1 / 2,1]): 1 \leq j \leq n\right\}$. If we define $h_{j}: g_{j} / \sum_{k=1}^{n} g_{k}$, then $\left\{h_{j}: 1 \leq j \leq n\right\}$ is a partition of unity subordinate to $\mathscr{U}$. Thus, $\mathscr{A} \aleph_{0}$-refines $\{F-G: G \in \mathscr{G}\}$. By Lemma 1 (i), this proves that

$$
d_{X}\left(F,\langle\mathscr{G}, \mathscr{A}\rangle_{\aleph_{0}}\right)=\sup \left\{d_{S}(F, \mathscr{G}): S \in \mathscr{A}(X)\right\} .
$$

In the sequel we prove that the supremum in the equality above is, in fact, a maximum. 
We identify each element $S$ in $\mathscr{A}(X)$ with a singleton in order to obtain a space $\bar{X}$ as follows. Since every function belonging to $\mathscr{A}$ is constant on each element $S$ in $\mathscr{A}(X)$, for every $h \in \mathscr{A}$ we can define a map $\bar{h}: \bar{X} \rightarrow \mathbb{R}$ by $\bar{h}(S)=h\left(x_{S}\right)$ for all $S \in \mathscr{A}(X)$, where $x_{S}$ is an arbitrary point of $S$. Denote by $\bar{A}$ the algebra on $\bar{X}$ formed by all the functions $\bar{h}$ with $h \in \mathscr{A}$. Then we endow $\bar{X}$ with the weak topology induced by the functions in $\overline{\mathscr{A}}$. It is clear that $\bar{X}$ is a Tychonoff space which is a continuous image of $X$ by the identification map. Hence $\bar{X}$ is a compact space.

Now, for every $G \in \mathscr{G}$, we define the map $L_{G}: \bar{X} \rightarrow \mathbb{R}^{+}$by $L_{G}(S)=d_{S}(F, G)=$ $\sup \{\|F(x)-G(x)\|: x \in S\}$ (we identify the elements of $\bar{X}$ with those of $\mathscr{A}(X)$ ). Let us see that $L_{G}$ is upper-semicontinuous on $\bar{X}$.

Let $\left\{S_{\delta}\right\}_{\delta \in D}$ be a net on $\bar{X}$ which converges to an arbitrary point $S_{0}$ of $\bar{X}$. Consider $U_{\epsilon}=(F-G)^{-1}\left(B_{\epsilon}\left(\left\{(F-G)\left(S_{0}\right)\right\}\right)\right)$ which is an open subset of $X$. In the first part of the proof we have proved that there is a function $h \in \mathscr{A}$ such that $h\left(S_{0}\right)=1$, $h\left(X \backslash U_{\epsilon}\right)=0$ and $0 \leq h \leq 1$. On the other hand $h$ defines a continuous map $\bar{h}$ on $\bar{X}$ with $\bar{h}\left(S_{0}\right)=1$. Thus there is $\delta_{0} \in D$ such that $\bar{h}\left(S_{\delta}\right)>1 / 2$ for all $\delta>\delta_{0}$. Hence if $\delta>\delta_{0}$ and $x \in S_{\delta}$ we have $h(x)>1 / 2$; this implies that $x \in U_{\epsilon}$. Thus $S_{\delta} \subseteq U_{\epsilon}$ for all $\delta>\delta_{0}$ or, equivalently, $(F-G)\left(S_{\delta}\right) \subseteq B_{\epsilon}\left(\left\{(F-G)\left(S_{0}\right)\right\}\right)$ for all $\delta>\delta_{0}$. Therefore $\|F(x)-G(x)\| \leq \sup \left\{\|F(y)-G(y)\|: y \in S_{\delta}\right\}+\epsilon$, for all $x \in S_{\delta}$ and for all $\delta>\delta_{0}$. From the inequality above we deduce that $L_{G}\left(S_{\delta}\right)=d_{S_{\delta}}(F, G) \leq d_{S_{0}}(F, G)+\epsilon=L_{G}\left(S_{0}\right)+\epsilon$, for all $\delta>\delta_{0}$. This proves that $L_{G}$ is upper-semicontinuous.

Now consider $L: \bar{X} \rightarrow \mathbb{R}^{+}$defined by $L(S)=d_{S}(F, \mathscr{G})=\inf \left\{L_{G}(S): G \in \mathscr{G}\right\}$. Note that $L$ is the infimum of a family of upper-semicontinuous maps, hence $L$ is upper-semicontinuous on the compact space $\bar{X}$. Therefore there is $S_{0} \in \bar{X}$ such that $L\left(S_{0}\right)=\sup \{L(S): S \in \bar{X}\}$. Thus, the supremum in the expression (*) is, in fact, a maximum and this completes the proof.

REMARK. If $\mathscr{G}$ is an $\mathscr{A}$-module and $\mathscr{A}$ is a subalgebra of $C(X)$ which contains the unit then $\langle\mathscr{G}, \mathscr{A}\rangle_{\aleph_{0}}=\mathscr{G}$. In this case the theorem above says that there is $S_{0} \in \mathscr{A}(X)$ such that $d_{X}(F, \mathscr{G})=d_{S_{0}}(F, \mathscr{G})$.

The following result is Machado's Theorem. Note that, in the proof, only Lemma 1, Lemma 2 and Theorem 5 are needed. In the sequel, if $\mathscr{A}$ is subalgebra of $C(X, \mathbb{C})$, we shall denote by $\mathscr{A}_{\mathbb{R}}$ the subalgebra of $\mathscr{A}$ formed by the real-valued functions in $\mathscr{A}$.

THEOREM 6. (Machado) For a compact space $X$, let $\mathscr{A}$ be a subalgebra of $C(X, \mathbb{C})$ which contains the unit and let $\mathscr{G}$ be a vector subspace of $C(X, E)$ which is an $\mathscr{A}$ module over $\mathscr{A}$. If $F \in C(X, E)$, then there is $S \in \mathscr{A}(X)$ such that $d_{X}(F, \mathscr{G})=$ $d_{S}(F, \mathscr{G})$. 
PROOF. Let $\mathscr{S}$ be the collection of all closed subsets of $X$ such that $S \subseteq X$ belongs to $\mathscr{S}$ if there is $T \subseteq X$ verifying the following properties:

(i) $S \subseteq T$,

(ii) $S$ is an antisymmetric set for $\left(\mathscr{A}_{\left.\right|_{T}}\right)_{\mathbb{R}}$,

(iii) $d_{S}(F, \mathscr{G})=d_{X}(F, \mathscr{G})=r$.

It is clear that $\mathscr{S}$ is non empty since $\mathscr{A}_{\mathbb{R}}$ has, by the theorem above, an antisymmetric set verifying (iii). Now we can order $\mathscr{S}$ in the following way: if $S$ and $T$ belong to $\mathscr{S}$ we say that $T \leq S$ when $S \subseteq T$ and $S$ is an antisymmetric set for $\left(\mathscr{A}_{\left.\right|_{T}}\right)_{\mathbb{R}}$. Let us see that every chain in $(\mathscr{S}, \leq)$ has an upper bound in $\mathscr{S}$. Suppose that $\left\{S_{i}: i \in I\right\}$ is a chain in $\mathscr{S}$, and let $S=\bigcap\left\{S_{i}: i \in I\right\}$. We have that $S$ belongs to $\mathscr{S}$; if not $d_{S}(F, \mathscr{G})<r$, that is, there is $G \in \mathscr{G}$ with $d_{S}(F, G)<r$. Let $0<r_{1}<r$ be such that $d_{S}(F, G)<r_{1}$ and let $U=\left\{x \in X:\|F(x)-G(x)\|<r_{1}\right\}$, then $S \subseteq U$ and, by compactness, there is $i \in I$ such that $S_{i} \subseteq U$, which is a contradiction. Thus $S$ belongs to $\mathscr{S}$ and is an upper bound for the chain. By Zorn's Lemma, there is a maximal element $B$ in $\mathscr{S}$. Now, $B$ must be an antisymmetric set for $\mathscr{A}$. If not $\left(\mathscr{A}_{\left.\right|_{B}}\right)_{\mathbb{R}}$ would have an antisymmetric subset properly included in $B$, which is a contradiction. Therefore $B$ is an $\mathscr{A}$-antisymmetric set and this completes the proof.

The following result is an application of the ideas above to realcompact spaces.

THEOREM 7. (Katětov) If $X$ is a paracompact space which is not realcompact, then there is at least one locally finite open cover of $X$ whose cardinality is measurable.

PROOF. Since $X$ is not realcompact, there is a free real maximal ideal $M$ in $C(X)$. For every $x \in X$, as $M$ is free, there is $g_{x} \in M$ such that $g_{x} \geq 0$ on $X$ and $g_{x}(x)=1$ (see [6] ). On the other hand, in a paracompact space, every open cover of the space has a locally finite partition of unity subordinate to that cover. Hence, we can apply Lemma 1 to the space $X$ for $\mathscr{A}=C(X), \mathscr{G}=M$ and $F=1$. Thus, as $C(X)$ consists only of singletons, we have that

$$
d_{X}(1,\langle M, C(X)\rangle)=\sup \left\{d_{[x]}(1, M): x \in X\right\}=0 .
$$

That is, there is a locally finite partition of unity $\left\{h_{i}: i \in I\right\}$ in $C(X)$ and a family of positive functions $\left\{g_{i}: i \in I\right\}$ in $M$ such that $\sum_{i \in I} h_{i}(x) \cdot g_{i}(x)>1 / 2$ for all $x \in X$. Let us define the following measure on the discrete space $I$. For $J \subseteq I, \mu(J)=1$ if $\sum_{i \in J} h_{i} \cdot g_{i} \notin M$, and $\mu(J)=0$ otherwise. From the properties of real maximal ideals, we deduce (see [6]) that $\mu$ is a countably additive, $\{0,1\}$-valued measure such that $\mu(I)=1$ and $\mu(\{i\})=0$ for each $i \in I$. This proves that I has measurable cardinality. Therefore the collection $\left\{\operatorname{coz} h_{i}: i \in I\right\}$ defines a locally finite open cover of $X$ with measurable cardinality, and this completes the proof. 
We finish by giving a generalization of Tietze's Extension Theorem for vectorvalued continuous functions. This result solves a question left open by Blair in [3, p. 69]. First we shall need some terminology.

Let $X$ be a topological space, $E$ a Banach space and $A$ a subset of $X$. If $F \in C(A, E)$ we say that $F$ is $z$-embedded in $X$ (see [3] ) when for every closed subset $N$ in $E$, there is a zero-set $Z$ in $X$ such that $Z \cap A=F^{-1}(N)$. We say that $F$ is uniformly $z$-embedded in $\mathrm{X}$ when for every countable cover of $F(A)$ consisting of open balls of fixed radius, $\left\{U_{n}: n \in \mathbb{N}\right\}$, there is a countable family of cozero-sets in $X$, $\left\{C_{n}: n \in \mathbb{N}\right\}$, such that $C_{n} \cap A=F^{-1}\left(U_{n}\right)$ for all $n \in \mathbb{N}$, and $A$ is completely separated from $\bigcap\left\{X \backslash C_{n}: n \in \mathbb{N}\right\}$ (we say that two subsets of $X, A$ and $B$, are completely separated when there is $h \in C(X)$, with $0 \leq h \leq 1$, such that $h(A)=\{0\}$ and $h(B)=\{1\})$. In the following $E$ designates a separable Banach space and $K$ a convex closed subset of $E$.

LEMMA 8. Let $F \in C(A, K)$ which is uniformly z-embedded in $X$ and let $G \in$ $C(X, K)$ such that $d_{A}(F, G) \leq r$. Then, for every positive real number $\epsilon$ there is $H \in C(X, K)$ with $d_{A}(F, H) \leq \epsilon$ and $d_{X}(G, H) \leq r+3 \epsilon$.

PROOF. Since $K$ is separable, there is a sequence in $K,\left\{a_{n}: n \in \mathbb{N}\right\}$, such that $\bigcup\left\{B_{\epsilon}\left(a_{n}\right): n \in \mathbb{N}\right\} \supseteq K$. Let $U_{n}=F^{-1}\left(B_{\epsilon}\left(a_{n}\right)\right), V_{m}=G^{-1}\left(B_{\epsilon}\left(a_{m}\right)\right)$ and consider the covers $\mathscr{U}=\left\{U_{n}: n \in \mathbb{N}\right\}$ and $\mathscr{V}=\left\{V_{m}: m \in \mathbb{N}\right\}$ (note that $\mathscr{U}$ is a cover of $K$ but $\mathscr{V}$ is a cover of $X$ ). As $F$ is uniformly $z$-embedded in $X$, there is a countable family of cozero-sets in $X,\left\{W_{n}: n \in \mathbb{N}\right\}$, such that $W_{n} \cap A=U_{n}$ and $A$ is completely separated from $\bigcap\left\{X \backslash W_{n}: n \in \mathbb{N}\right\}$. Let $h \in C(X)$ such that $0 \leq h \leq 1, h(A)=\{0\}$ and $h\left(\bigcap\left\{X \backslash W_{n}: n \in \mathbb{N}\right\}\right)=\{1\}$; if we set $W_{0}=\operatorname{coz} h$, then $\mathscr{W}=\left\{W_{n}: n \in \mathbb{N} \cup\{0\}\right\}$ is an open cover of $X$ formed by cozero-sets. Let us take now the cover of $X$ defined by $\mathscr{V} \cap \mathscr{W}=\left\{V_{m} \cap W_{n}: m \in \mathbb{N}, n \in \mathbb{N} \cup\{0\}\right\}$. Since $\mathscr{V} \cap \mathscr{W}$ is a countable open cover of $X$ formed by cozero-sets, there is a locally finite partition of unity subordinate to the cover (see [1, Section 11]). Let $\left\{h_{i}: i \in \mathbb{N}\right\}$ be this partition of unity and let us define the function $H$ as follows, $H=\sum_{i \in \mathbb{N}} c_{i} . h_{i}$, where $c_{i}=a_{n(i)}$ if there are $n \in \mathbb{N}$ and $m \in \mathbb{N}$ verifying:

(1) $\operatorname{supp} h_{i} \subseteq V_{m} \cap W_{n}$;

(2) $V_{m} \cap W_{n} \cap A \neq \emptyset$.

Let $n(i)$ be the smallest natural number $n>0$ with this property. On the other hand, $c_{i}=a_{m(i)}$ when any pair $(m, n) \in \mathbb{N} \times \mathbb{N}$ fails to verify (1) and (2) above, and $m(i)$ is the smallest natural number $m$ such that $\operatorname{supp} h_{i} \subseteq V_{m}$. We have, since $K$ is a convex subset of $E$, that $H \in C(X, K)$.

For every $x \in A$ we have that

$$
\|F(x)-H(x)\|=\left\|\sum_{i \in \mathbb{N}} h_{i}(x) \cdot F(x)-\sum_{i \in \mathbb{N}} h_{i}(x) \cdot c_{i}\right\|
$$




$$
\leq \sum_{i \in \mathbb{N}} h_{i}(x) .\left\|F(x)-c_{i}\right\| .
$$

Now, if $x \in A$, it is clear that $c_{i}=a_{n(i)}$ for all $i \in \mathbb{N}$ such that $x \in A \cap\left(\operatorname{supp} h_{i}\right)$. Hence, when $x \in A$ and $h_{i}(x) \neq 0$, we have $c_{i}=a_{n(i)}$ and $x \in U_{n(i)}$; this implies that $\left\|F(x)-a_{n(i)}\right\|<\epsilon$. Thus $\|F(x)-H(x)\|<\epsilon$ for all $x \in A$ or, equivalently, $d_{A}(F, H) \leq \epsilon$.

Let us calculate now $d_{X}(F, G)$. For $x \in A$ we have $\|G(x)-H(x)\| \leq$ $\|G(x)-F(x)\|+\|F(x)-H(x)\|<r+\epsilon$. If $x \notin A$, then $\|G(x)-H(x)\| \leq$ $\sum_{i \in \mathbb{N}} h_{i}(x) .\left\|G(x)-c_{i}\right\|$. Let us suppose that $c_{i}=a_{m(i)}$, then $\left\|G(x)-a_{m(i)}\right\|<\epsilon$ for all $x \in X$ such that $h_{i}(x) \neq 0$. On the other hand, when $c_{i}=a_{n(i)}$, there is $m_{i} \in \mathbb{N}$ such that (1) $\operatorname{supp} h_{i} \subseteq V_{m_{i}} \cap W_{n(i)}$ and (2) $V_{m_{i}} \cap W_{n(i)} \cap A \neq \emptyset$. Choose $y \in V_{m_{i}} \cap U_{n(i)}$; then $\left\|G(y)-a_{m_{i}}\right\|<\epsilon$ and $\left\|F(y)-a_{n(i)}\right\|<\epsilon$. On the other hand, $\|G(y)-F(y)\|<r$ by hypothesis. Thus $\left\|a_{m_{i}}-a_{n(i)}\right\|<r+2 \epsilon$. Now, let $x \in X$ be such that $h_{i}(x) \neq 0$. Since $x \in V_{m_{i}}$ we have that $\left\|G(x)-a_{m_{i}}\right\|<\epsilon$, and consequently that $\left\|G(x)-a_{n(i)}\right\|<r+3 \epsilon$. Therefore $\|G(x)-H(x)\|<r+3 \epsilon$ for all $x \in X$. That is, $d_{X}(G, H) \leq r+3 \epsilon$.

THEOREM 9. Let $A$ be a subspace of the space $X$, and consider $F \in C(A, K)$ with $K$ a closed convex subset of a separable Banach space $E$. Then $F$ may be extended to a function in $C(X, K)$ if and only if $F$ is uniformly $z$-embedded in $X$.

ProOF. Given that $K$ is a metric space it is not hard to check necessity in the theorem. Let us show sufficiency.

Let $F \in C(X, K)$ be uniformly z-embedded in $X$. We define $F_{0} \in C(X, K)$ like an arbitrary constant value in $K$. By applying the lemma above we can get $F_{1} \in C(X, K)$ such that $d_{A}\left(F_{1}, F\right)<1 / 4$. Suppose we have defined $\left\{F_{1}, F_{2}, \ldots, F_{n}\right\} \subseteq C(X, K)$ such that $d_{A}\left(F_{j}, F\right)<1 / 4^{j}$, for $1 \leq j \leq n$, and $d_{X}\left(F_{j}, F_{j+1}\right)<1 / 4^{j-1}$, for $1 \leq j \leq$ $n-1$. By applying again the lemma above we can get $F_{n+1} \in C(X, K)$ such that $d_{A}\left(F_{n+1}, F\right)<1 / 4^{n+1}$ and $d_{X}\left(F_{n}, F_{n+1}\right)<1 / 4^{n}+3 / 4^{n+1}<1 / 4^{n-1}$, that is, $\left\{F_{n}\right\}_{n=1}^{\infty}$ is a sequence which converges uniformly to $F$ in $A$ and it is a Cauchy sequence in $C(X, K)$ for the uniform convergence topology. Let $\hat{F}$ be the uniform limit of $\left\{F_{n}\right\}$; then $\hat{F} \in C(X, K)$ and $\hat{F}_{\left.\right|_{A}}=F$. This completes the proof.

COROLLARY 10. Let $X$ be a normal space and $A$ a closed subspace of $X$. Then, for every closed convex subset $K$ of a separable Banach space $E$, we have that $C(X, K)_{\left.\right|_{A}}=C(A, K)$.

PROOF. This is clear by noting that every function in $C(A, K)$ is uniformly $z$ embedded in $X$ when $A$ is a closed subset of a normal space. 
REMARKS. With slight modifications in the proof of Lemma 8, we can generalize the corollary above when $X$ is a $\gamma$-collectionwise normal space and $K$ a $\gamma$-separable metrizable complete convex subset of a locally convex space $E$ such that the metric of $K$ is defined by a countable family of seminorms (see [1] for a comprehensive exposition of this topic). Also Theorem 9 may be generalized to a function with range in a closed convex subset of an arbitrary Banach space when $X$ is a paracompact space, if we consider the obvious generalization of uniformly $z$-embedded function.

\section{References}

[1] R. A. Alo and H. L. Shapiro, Normal topological spaces (Cambridge University Press, London, 1974).

[2] E. Bishop, 'A generalization of the Stone-Weierstrass Theorem', Pacific J. Math. 11 (1961), 777-783.

[3] R. L. Blair, 'Extensions of Lebesgue sets and of real-valued functions', Czechoslovak Math. J. 31 (1981), 63-74.

[4] R. B. Burckel, 'Bishop's Stone-Weierstrass Theorem', Amer. Math. Monthly 91 (1984), 22-32.

[5] D. A. Edwards, 'A short proof of a Theorem of Machado', Math. Proc. Cambridge Philos. Soc. 99 (1986), 111-114.

[6] L. Gillman and M. Jerison, Rings of continuous functions (Van Nostrand, Princeton, 1960).

[7] M. Katětov, 'Measures in fully normal spaces', Fund. Math. 38 (1951), 73-84.

[8] S. Machado, 'On Bishop's generalization of the Stone-Weierstrass Theorem', Indag. Math. 39 (1977), 218-224.

[9] S. Mrowka, 'On some approximation theorems', Nieuw Arch. Wisk. 16 (1968), 94-111.

[10] T. J. Ransford, 'A short elementary proof of the Stone-Weierstrass-Bishop Theorem', Math. Proc. Cambridge Philos. Soc. 96 (1984), 309-311. 